\title{
Hybridization-related correction to the jellium model for fullerenes
}

\author{
A V Verkhovtsev ${ }^{1,2}, \mathbf{R}$ G Polozkov ${ }^{2}, \mathrm{~V}$ K Ivanov $^{2}$, A V Korol $^{1}$, \\ A V Solov'yov ${ }^{1} \pm$ \\ ${ }^{1}$ Frankfurt Institute for Advanced Studies, Ruth-Moufang-Str. 1, 60438 Frankfurt \\ am Main, Germany \\ ${ }^{2}$ St. Petersburg State Polytechnic University, Politekhnicheskaya ul. 29, 195251 St. \\ Petersburg, Russia \\ E-mail: verkhovtsev@fias.uni-frankfurt.de
}

\begin{abstract}
We introduce a new type of correction for a more accurate description of fullerenes within the spherically symmetric jellium model. This correction represents a pseudopotential which originates from the comparison between an accurate ab initio calculation and the jellium model calculation. It is shown that such a correction to the jellium model allows one to account, at least partly, for the $\mathrm{sp}^{2}$-hybridization of carbon atomic orbitals. Therefore, it may be considered as a more physically meaningful correction as compared with a structureless square-well pseudopotential which has been widely used earlier.

PACS numbers: 31.15.A-, 31.15.xr, 36.40.-c
\end{abstract}

\section{Introduction}

Since the discovery of fullerenes by Kroto et al. [1] in 1985, these molecules have been the objects of intensive experimental and theoretical investigations (see, e.g., [2]). At present, the investigation of fullerenes is active since they are proposed to be used in various fields of science and technology. For instance, excitation of fullerenes, placed in a biological medium, by an external radiation or incident heavy ions may lead to an active generation of secondary electrons or reactive oxygen species. This allows fullerenes to be potentially used as sensitizers in photodynamic therapy [3]. A very important fundamental problem closely related to the aforementioned application is an adequate description of dynamic response of fullerenes to external fields or to the interaction with projectiles. Processes of scattering of electrons, photons and heavy charged particles on various atomic clusters and fullerenes, in particular, have been actively studied during the past several decades (see, e.g., the review [4] and references therein). Not the least of the factors for a proper description of the dynamic response

$\ddagger$ On leave from A.F. Ioffe Physical-Technical Institute, St. Petersburg, Russia 
of a many-electron system is an adequate description of the ground- and excited-state (including excitation into continuum) properties of the system under study.

Contemporary software for quantum-chemical calculations (e.g., Gaussian 09 [5]) provides an accurate quantitative description of the ground state of manyparticle systems (fullerenes, in particular), and allows one to obtain information on geometrical and chemical properties of the system. However, the description of dynamic properties, which play an important role in the process of photoionization, by means of such programs faces significant difficulties. Dynamic properties (e.g., dynamic polarizability) are closely related to the response of a many-electron system to an external electromagnetic field. In many cases, the properties are governed by a collective excitation of electrons and the formation of plasmon resonances in the excitation spectra [6]. In various systems plasmon resonances lie either below the ionization threshold (in metal clusters) or above it (e.g., in fullerenes). Out of these two classes of atomic clusters, only the optical response of metal clusters has been calculated so far with the help of quantum-chemical programs (see, e.g. [7, 8]). Collective electron excitations in fullerenes, which lie in the continuous spectrum, have not been described so far by means of quantum-chemical programs. However, this can be achieved within simplified model approximations. A minimum requirement is that these approximations must provide an accurate quantitative description of the ground-state features of the systems under study, in order to be applied to the investigation of the dynamic response and to the calculation of the photoabsorption (or, in particular, photoionization) spectrum.

One of the well-known and widely used approaches is based on the jellium model [9]. It was applied frequently to the description of ground-state properties of metal clusters [911] and fullerenes [12,13], as well as to the investigation of photoexcitation processes arising in these systems [13 21].

In [12, 22], it was stated that the ground-state properties of fullerenes cannot be described properly by the standard jellium model which produces, in particular, unreliable values for the total energy [12]. To avoid this, adding of structureless pseudopotential corrections was suggested [12]. As a rule, a phenomenological squarewell (SW) pseudopotential has been commonly used in the calculations [12, 16, 18, 21]. It was claimed that accounting for such a pseudopotential increases the accuracy of the jellium-based description [16] and, for instance, allows one to reproduce the experimental value of the first ionization potential of $\mathrm{C}_{60}$ [21]. Nonetheless, the applicability of the jellium model for fullerenes and the choice of parameters of the used SW pseudopotential have not been clearly justified so far from a physical viewpoint.

In this paper, we use another methodology and introduce a structured pseudopotential which originates from the comparison of an accurate $a b$ initio calculation with the jellium-based one. Using this pseudopotential as a correction to the standard jellium model, one can introduce effects of the $\mathrm{sp}^{2}$-hybridization of carbon atomic orbitals into the jellium model and relate parameters of the model with the features of the system obtained from the more precise calculation. By means of the presented pseudopotential, a relatively simple jellium model acquires more physical sense 
and parameters of the model obtain a clear physical justification. Hereby, we confirm the relevance of using the jellium model for the description of fullerenes. Investigating two molecules, $\mathrm{C}_{60}$ and $\mathrm{C}_{20}$, we show that the results obtained have a common origin and that they could be also extended to other highly symmetric fullerenes.

The atomic system of units, $m_{e}=|e|=\hbar=1$, is used throughout the paper.

\section{Methods of investigation}

\subsection{Jellium model}

In this paper, the fullerenes $\mathrm{C}_{60}$ and $\mathrm{C}_{20}$ are treated within the jellium model which is based on an assumption that a many-electron system is considered as a sum of two interacting subsystems: a valence electrons subsystem and a positively charged ionic core. One of the stable isomers of $\mathrm{C}_{20}$ corresponds geometrically to the regular dodecahedron [23] and, like the truncated icosahedron $\mathrm{C}_{60}$, has the symmetry of the $I_{h}$ point group which is very close to spherical symmetry. Therefore, a detailed ionic structure of the systems under study is substituted by the uniform spherically symmetric distribution of the positive charge, in the field of which the motion of the valence electrons is considered [9].

The valence $2 \mathrm{~s}^{2} 2 \mathrm{p}^{2}$ electrons in each carbon atom form a cloud of delocalized electrons, while the inner-shell $1 \mathrm{~s}^{2}$ electrons are treated as frozen and not taken into consideration. Thereby, we consider 240 delocalized electrons in $\mathrm{C}_{60}$ and 80 electrons in $\mathrm{C}_{20}$. The valence electrons are moving in a spherically symmetric central field, so one can construct the electronic configuration described by the unique set of quantum numbers $\{n, l\}$ where $n$ and $l$ are the principal and orbital quantum numbers, respectively.

Since it is commonly acknowledged [24 26] that $\mathrm{C}_{60}$, as well as other fullerenes, is formed from fragments of planar graphite sheets, it is natural to match the $\sigma$ - and $\pi$-orbitals of graphite to the nodeless and the single-node wavefunctions of a fullerene, respectively [27]. Carbon atoms within a graphite sheet are connected by $\sigma$-bonds, whereas different sheets are connected by $\pi$-bonds. In the fullerene, the nodeless $\sigma$ orbitals are localized at the radius of the ionic core while the single-node $\pi$-orbitals are oriented perpendicularly to the fullerene surface. The ratio of $\sigma$ - to $\pi$-orbitals in $\mathrm{C}_{60}$ should be equal to $3: 1$ due to the $\mathrm{sp}^{2}$-hybridization of carbon orbitals [28]. Thereby, the electronic configuration of the delocalized electrons in $\mathrm{C}_{60}$ is written in the form [13]:

$$
\begin{gathered}
1 \mathrm{~s}^{2} 2 \mathrm{p}^{6} 3 \mathrm{~d}^{10} 4 \mathrm{f}^{14} 5 \mathrm{~g}^{18} 6 \mathrm{~h}^{22} 7 \mathrm{i}^{26} 8 \mathrm{k}^{30} 9 \mathrm{l}^{34} 10 \mathrm{~m}^{18} \\
2 \mathrm{~s}^{2} 3 \mathrm{p}^{6} 4 \mathrm{~d}^{10} 5 \mathrm{f}^{14} 6 \mathrm{~g}^{18} 7 \mathrm{~h}^{10}
\end{gathered}
$$

Radial wavefunctions of the $1 \mathrm{~s} \ldots 10 \mathrm{~m}$ shells are nodeless, while the wavefunctions of the $2 \mathrm{~s} \ldots 7 \mathrm{~h}$ shells have one radial node each.

Using the same methodology, one defines the electronic configuration of the 80 delocalized electrons in $\mathrm{C}_{20}$ as follows:

$$
1 \mathrm{~s}^{2} 2 \mathrm{p}^{6} 3 \mathrm{~d}^{10} 4 \mathrm{f}^{14} 5 \mathrm{~g}^{18} 6 \mathrm{~h}^{10} 2 \mathrm{~s}^{2} 3 \mathrm{p}^{6} 4 \mathrm{~d}^{10} 5 \mathrm{f}^{2} .
$$


Within the jellium model the fullerene core of the charged carbon ions, $\mathrm{C}^{4+}$, is described as a positively charged spherical layer of a finite thickness $\Delta R=R_{2}-R_{1}$. The thickness $\Delta R$ is chosen to be equal to $1.5 \AA$ which corresponds to a typical diameter of a carbon atom [29] and refers to experimental data from [18]. The potential of the core may be written as:

$$
U_{\text {core }}(r)=-N \times\left\{\begin{array}{cl}
\frac{3}{2} \frac{R_{2}^{2}-R_{1}^{2}}{R_{2}^{3}-R_{1}^{3},} & r<R_{1} \\
\frac{1}{2\left(R_{2}^{3}-R_{1}^{3}\right)}\left(3 R_{2}^{2}-r^{2}\left(1+\frac{2 R_{1}^{3}}{r^{3}}\right)\right), & R_{1} \leq r \leq R_{2} \\
\frac{1}{r}, & r>R_{2}
\end{array}\right.
$$

where $N$ is the number of delocalized electrons in a fullerene $\left(N=240\right.$ in $\mathrm{C}_{60}$ and $N=80$ in $\left.\mathrm{C}_{20}\right), R_{1}=R-\Delta R / 2$ and $R_{2}=R+\Delta R / 2$ with $R$ standing for a fullerene radius $\left(R_{\mathrm{C}_{60}}=3.54 \AA\right.$ and $R_{\mathrm{C}_{20}}=2.04 \AA[30]$ ).

The electronic subsystem is treated within the local density approximation (LDA). Single-electron wave functions $\phi_{n l m}(\mathbf{r})$ and the corresponding energies $\varepsilon_{n l}$ are determined from a system of self-consistent Kohn-Sham equations:

$$
\begin{aligned}
& {\left[-\frac{\Delta}{2}+U_{\text {eff }}(\mathbf{r})\right] \phi_{n l m}(\mathbf{r})=\varepsilon_{n l} \phi_{n l m}(\mathbf{r}),} \\
& U_{\text {eff }}(\mathbf{r})=U_{\text {core }}(r)+\int \frac{\rho\left(\mathbf{r}^{\prime}\right)}{\left|\mathbf{r}-\mathbf{r}^{\prime}\right|} d \mathbf{r}^{\prime}+U_{\mathrm{XC}}^{\mathrm{LDA}}(\mathbf{r}), \\
& \rho(\mathbf{r})=\sum_{n l} \sum_{m=-l}^{l} \frac{N_{n l}}{2(2 l+1)}\left|\phi_{n l m}(\mathbf{r})\right|^{2},
\end{aligned}
$$

where $N_{n l}$ is a number of electrons in the $n l$-shell. The exchange-correlation potential $U_{\mathrm{XC}}^{\mathrm{LDA}}(\mathbf{r})$ is represented as a sum of the Slater exchange potential and a correlation potential:

$$
U_{\mathrm{XC}}^{\mathrm{LDA}}(\mathbf{r})=U_{\mathrm{X}}(\mathbf{r})+U_{\mathrm{C}}(\mathbf{r})=-\left(\frac{3}{\pi}\right)^{1 / 3} \rho^{1 / 3}(\mathbf{r})+U_{\mathrm{C}}(\mathbf{r}) .
$$

In the calculations, we used Perdew and Zunger parameterization of the correlation potential [31] which is presented in the form

$$
\begin{aligned}
& U_{\mathrm{C}}\left(r_{s}\right)=\varepsilon_{\mathrm{C}}\left(r_{s}\right) \frac{1+1.229 \sqrt{r_{s}}+0.444 r_{s}}{1+1.053 \sqrt{r_{s}}+0.333 r_{s}}, \\
& \varepsilon_{\mathrm{C}}\left(r_{s}\right)=-\frac{0.142}{1+1.053 \sqrt{r_{s}}+0.333 r_{s}}
\end{aligned}
$$

where $r_{s}(\mathbf{r})=(4 \pi \rho(\mathbf{r}) / 3)^{-1 / 3}$ is the local Wigner-Seitz radius for the electronic subsystem and $\varepsilon_{\mathrm{C}}\left(r_{s}\right)$ is the correlation energy per electron. 


\subsection{Ab initio calculations}

The ab initio calculations were performed using the Gaussian 09 package [5]. For the description of the $\mathrm{C}_{60}$ and $\mathrm{C}_{20}$ fullerenes we used the split-valence triple-zeta basis set $6-311+\mathrm{G}(\mathrm{d})$ with an additional set of polarization and diffuse functions. The systems were calculated by means of the density functional theory. To account for the exchange and correlation corrections, the Slater exchange functional [32] and the local Perdew functional (the so-called Perdew Local, PL) [31] were used. By applying these rather simple functionals we wanted to achieve a full similarity in the description of the electronic subsystem within the jellium model and the $a b$ initio approaches.

The total electrostatic potential of the system is represented as a sum of the nuclear and electronic parts:

$$
U_{\mathrm{tot}}(\mathbf{r})=U_{\mathrm{n}}(\mathbf{r})+U_{\mathrm{el}}(\mathbf{r})=-\sum_{A} \frac{Z_{A}}{\left|\mathbf{r}-\mathbf{R}_{A}\right|}+\int \frac{\rho\left(\mathbf{r}^{\prime}\right)}{\left|\mathbf{r}-\mathbf{r}^{\prime}\right|} d \mathbf{r}^{\prime}
$$

The electron density $\rho(\mathbf{r})$ and the potential $U_{\mathrm{n}}(\mathbf{r})$ created by all carbon ions, $\mathrm{C}^{4+}\left(1 \mathrm{~s}^{2}\right)$, were extracted from the Gaussian output file with the help of the Multiwfn software 33. The potential $U_{\mathrm{el}}(\mathbf{r})$ created by the delocalized electrons was calculated separately using the extracted electron density.

The jellium model treats the fullerenes $\mathrm{C}_{60}$ and $\mathrm{C}_{20}$ as spherically symmetric objects while a more precise $a b$ initio calculation accounts for the real icosahedral symmetry of the molecules. Therefore, to draw an analogy between the two methods we averaged the exact electrostatic potential and the electron density over the directions of the position vector $\mathbf{r}$ :

$$
\begin{aligned}
& \bar{U}_{\mathrm{tot}}(r)=\bar{U}_{\mathrm{n}}(r)+\bar{U}_{\mathrm{el}}(r), \\
& \bar{U}_{\mathrm{i}}(r)=\frac{1}{4 \pi} \int U_{\mathrm{i}}(\mathbf{r}) d \Omega \quad(\mathrm{i}=\text { tot }, \mathrm{n}, \mathrm{el}), \\
& \bar{\rho}(r)=\frac{1}{4 \pi} \int \rho(\mathbf{r}) d \Omega .
\end{aligned}
$$

The averaged electron density includes only delocalized electrons, while the inner electron orbitals are excluded from the consideration.

\section{Numerical results}

In this section, we compare the results of the $a b$ initio and the jellium model calculations for $\mathrm{C}_{60}$ and $\mathrm{C}_{20}$. The fullerene $\mathrm{C}_{60}$ is discussed in detail below. The results for the $\mathrm{C}_{20}$ molecule and the comparison with $\mathrm{C}_{60}$ are discussed further in this section.

Using the methodology implemented in a number of papers [12, 16, 18, 21], we add a negative SW pseudopotential $U_{\mathrm{SW}}$ to the core potential (1):

$$
U_{\text {core }}(r) \rightarrow\left\{\begin{array}{cl}
U_{\text {core }}(r)+U_{\mathrm{SW}} & , R_{1} \leq r \leq R_{2} \\
U_{\text {core }}(r) & , \text { otherwise }
\end{array}\right.
$$


The depth of the SW potential was chosen to obtain the same value of the outer-shell ionization potential as the defined one from the quantum-chemical calculation. The pseudopotential $U_{\mathrm{SW}}$ is shown by the dashed red curve in the lower panel of figure 3 .

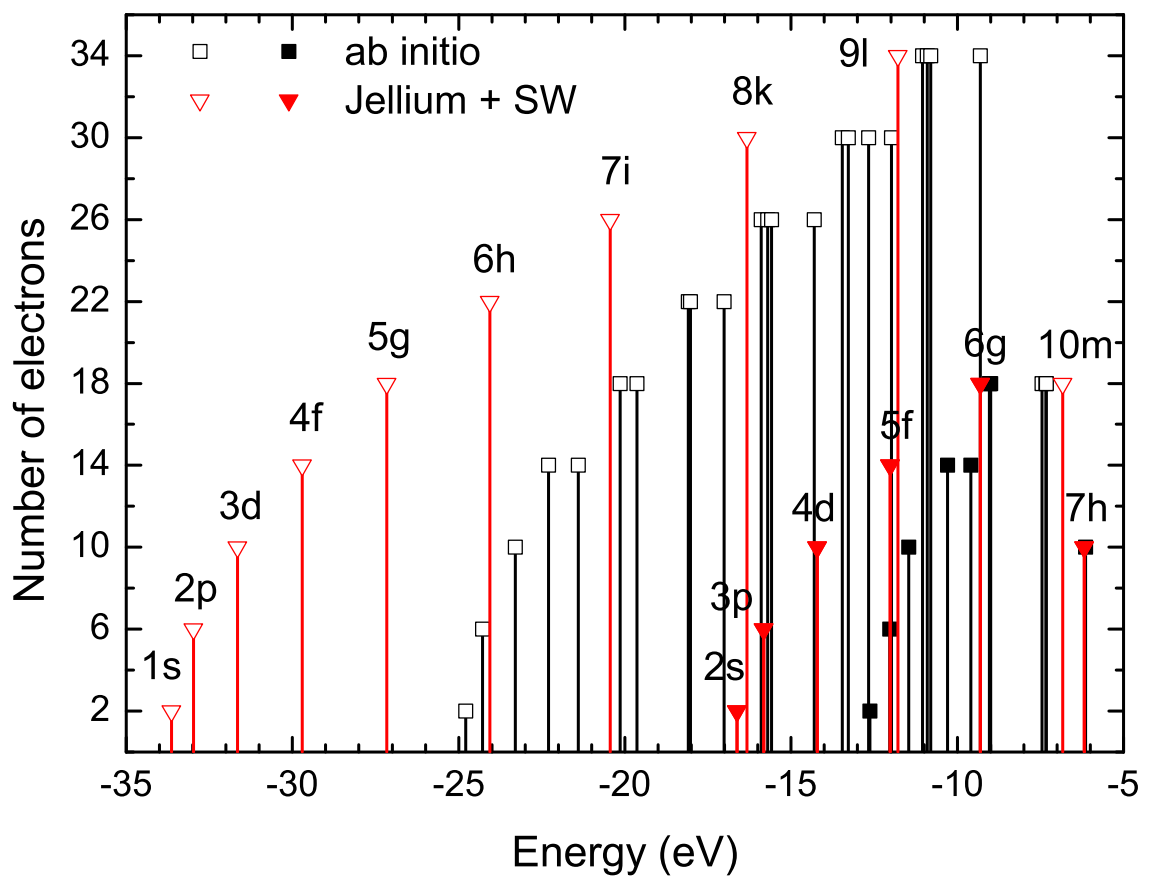

Figure 1. Single-electron energy levels of $\mathrm{C}_{60}$ obtained from the ab initio calculation (empty and filled squares) and within the jellium model with an additional square-well (SW) pseudopotential (empty and filled triangles). Nodeless $\sigma$-orbitals and single-node $\pi$-orbitals are labeled by empty and filled symbols, respectively.

Single-electron energy spectra obtained from the $a b$ initio calculation and within the jellium model are presented in figure 1, For the ease of perception, the height of levels of split stated (black lines) corresponds to the occupation of shells within the jellium model (red lines). Ionization potentials of several outer shells (6g, $10 \mathrm{~m}$ and $7 \mathrm{~h}$ ) are in a good agreement with the $a b$ initio results while the remainder of the jellium spectrum is significantly broadened and differs from the more precise calculation. It should be mentioned that none of the various jellium-based calculations of $\mathrm{C}_{60}$ performed earlier [12,13, 16, 17,20,21] can produce the quantitative agreement of the single-electron spectrum with that one obtained from the more precise ab initio calculation.

The radial density of the delocalized electrons obtained within the two approaches is presented in figure 2. It is shown that the standard jellium model without any corrections (dashed red curve) fails to represent the results of the ab initio calculation (black curve). The additional SW pseudopotential does not modify the density distribution significantly (solid red curve).

As shown in figures 1 and 2, the jellium model with a simple additional 


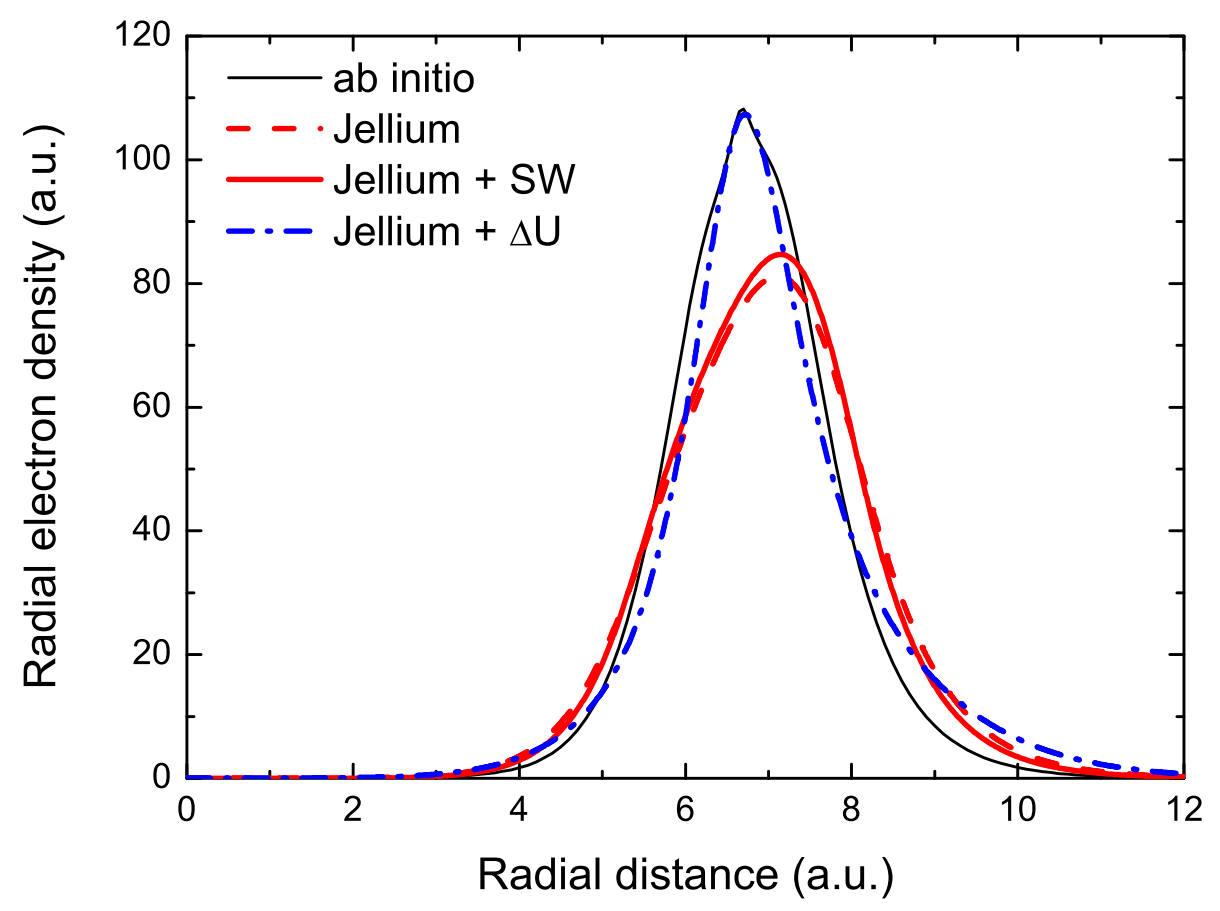

Figure 2. Radial electron density of $\mathrm{C}_{60}$ obtained from the ab initio calculation (solid black curve) and calculated by means of the jellium model: the standard one (dashed red curve), with the additional SW pseudopotential (solid red curve) and with the additional pseudopotential $\Delta U$ (dash-dotted blue curve).

pseudopotential represents neither the single-electron energy spectrum nor the electron density distribution. As opposed to more precise quantum chemistry methods, the jellium model does not take into account chemical features of the fullerene, such as hybridization of atomic orbitals in the formation of chemical bonding. However, the jellium model can be improved by means of a more sophisticated pseudopotential which will allow one to describe chemical properties of the real system. In this paper, we introduce the correction as a difference between the total electrostatic potential of the system obtained from the ab initio calculation and the one obtained within the jellium model:

$$
\Delta U(r)=U_{\text {tot }}^{\mathrm{QC}}(r)-U_{\text {tot }}^{\mathrm{jel}}(r),
$$

where $U_{\text {tot }}^{\mathrm{QC}}(r)$ is defined by Eq. (8), and the potential $U_{\mathrm{tot}}^{\mathrm{jel}}(r)$ obtained within the jellium model is defined as:

$$
U_{\text {tot }}^{\text {jel }}(r)=U_{\text {core }}(r)+\int \frac{\rho\left(\mathbf{r}^{\prime}\right)}{\left|\mathbf{r}-\mathbf{r}^{\prime}\right|} d \mathbf{r}^{\prime} .
$$

The total potentials $U_{\text {tot }}^{\mathrm{QC}}(r)$ and $U_{\mathrm{tot}}^{\mathrm{jel}}(r)$ of $\mathrm{C}_{60}$ as well as their difference $\Delta U(r)$ are shown in figure 3 , 
We note that previously several spiritually close averaged pseudopotentials have been introduced to correct the jellium model. For instance, it was done in the case of inhomogeneous electron gas on metal surfaces [34,35] and for spherically symmetric metallic clusters [36, 37].

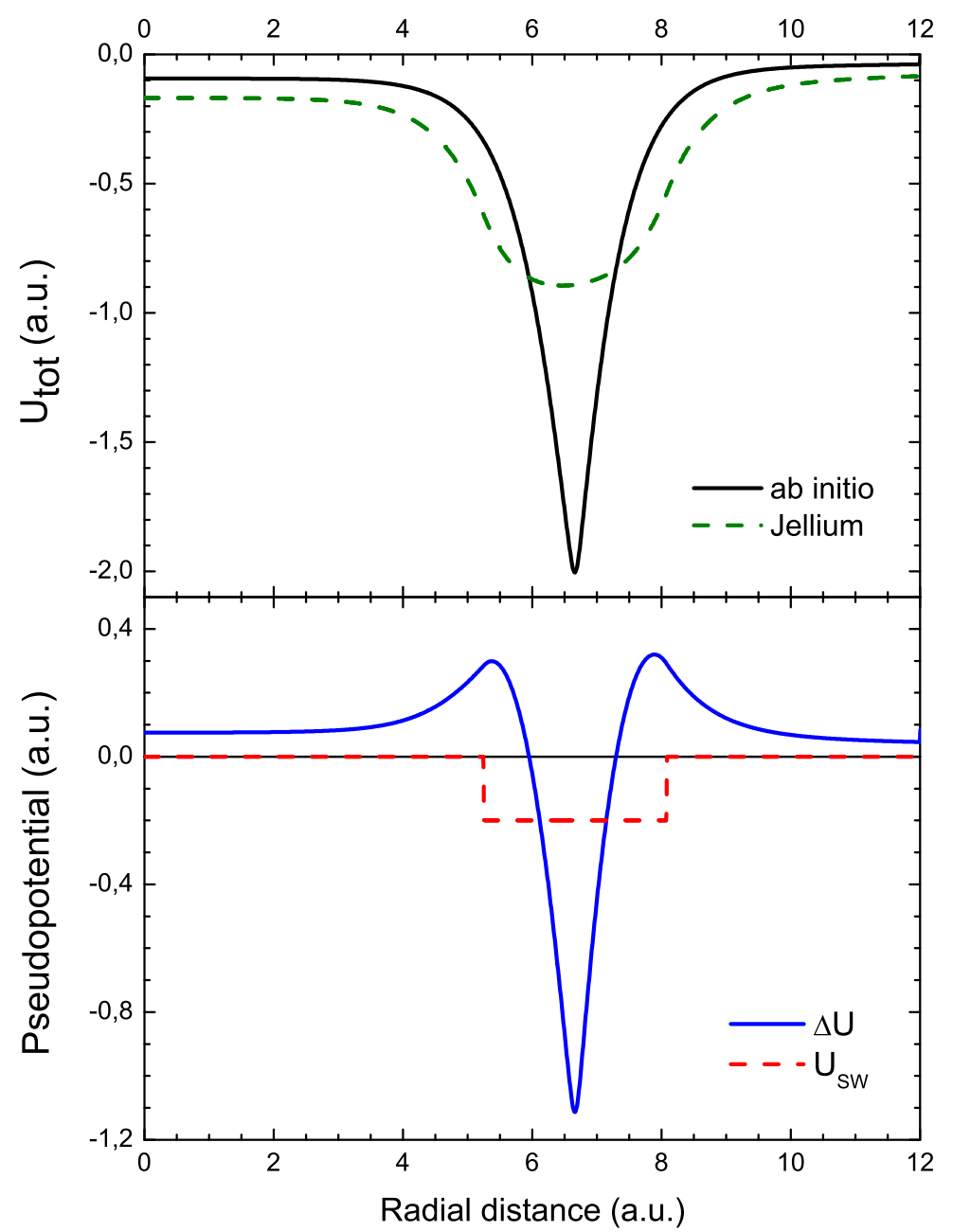

Figure 3. Upper panel: total electrostatic potential of $\mathrm{C}_{60}$ obtained from the ab initio quantum chemistry calculation (solid curve) and within the jellium model (dashed curve). Lower panel: the difference $\Delta U$ between the total electrostatic potential of $\mathrm{C}_{60}$ calculated by the $a b$ initio methods and the one calculated within the jellium model (solid blue curve). The SW pseudopotential $U_{\mathrm{SW}}$ is also shown for the comparison (dashed red curve).

As opposed to the SW pseudopotential which affects equally all electrons of the system, $\Delta U$ is an alternating-sign pseudopotential (see the lower panel of figure 3), therefore it is attractive in the vicinity of the fullerene ionic core and repulsive at larger distances from the fullerene surface. This means that such a potential affects differently the $\sigma$ - and $\pi$-electrons of $\mathrm{C}_{60}$ which are located on the surface of the molecule and perpendicularly to it, respectively. Therefore, one can conclude that by means of a such 
potential it is possible to account, to some extent, for the hybridization properties of the fullerene.

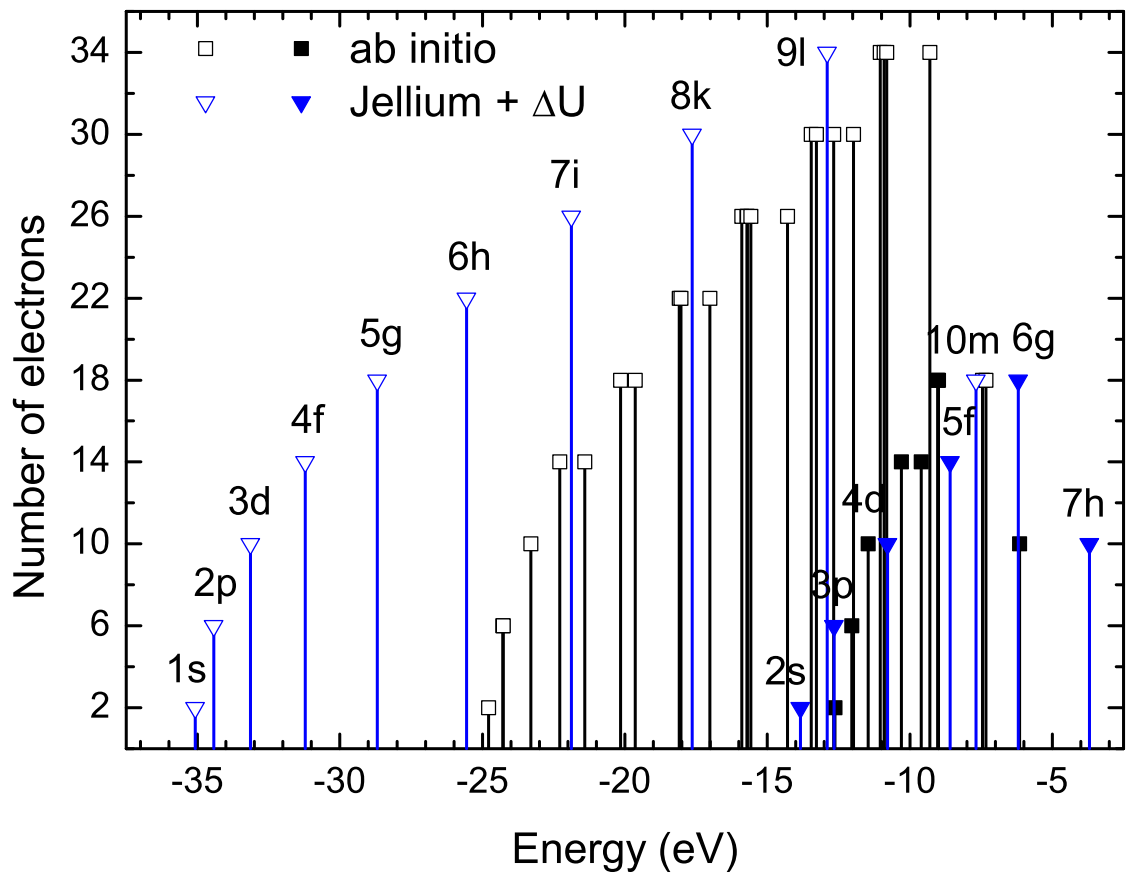

Figure 4. Single-electron energy levels of $\mathrm{C}_{60}$ obtained from the ab initio calculation (empty and filled squares) and within the modified jellium model with an additional pseudopotential $\Delta U$ (empty and filled triangles). Nodeless $\sigma$-orbitals and single-node $\pi$-orbitals are labeled by empty and filled symbols, respectively.

The single-electron energy spectrum obtained within the "modified" jellium model with $\Delta U$ taken as an additional pseudopotential is presented in figure 4. The modification allows one to obtain a better agreement of the jellium calculation with the $a b$ initio one for the inner single-node $2 \mathrm{~s} \ldots 5 \mathrm{f}$ orbitals. On the contrary, it shifts the $6 \mathrm{~g}$ and $7 \mathrm{~h}$ ionization potentials by 2.8 and $2.5 \mathrm{eV}$, respectively, and still does not lead to a better quantitative agreement for the whole spectrum (see figure 4).

Introduction of the alternating-sign pseudopotential $\Delta U$ allows one to improve significantly the electron density distribution (see the dash-dotted blue curve in figure 2). The difference between the $a b$ initio calculated electron density and the one from the jellium model calculation in the spatial region $8-12$ a.u. may contribute to the shift of $6 \mathrm{~g}$ and $7 \mathrm{~h}$ ionization potentials (see figure 4).

Below we present and discuss the results for the $\mathrm{C}_{20}$ molecule. Following the formalism described above for $\mathrm{C}_{60}$, the additional pseudopotential $\Delta U$ is introduced as a difference between the total electrostatic potential of $\mathrm{C}_{20}$ obtained from the $a b$ initio quantum-chemical calculation and the one obtained within the jellium model. Figure 5 represents the correction $\Delta U$ calculated for $\mathrm{C}_{60}$ and $\mathrm{C}_{20}$. It is shown that $\Delta U$ 


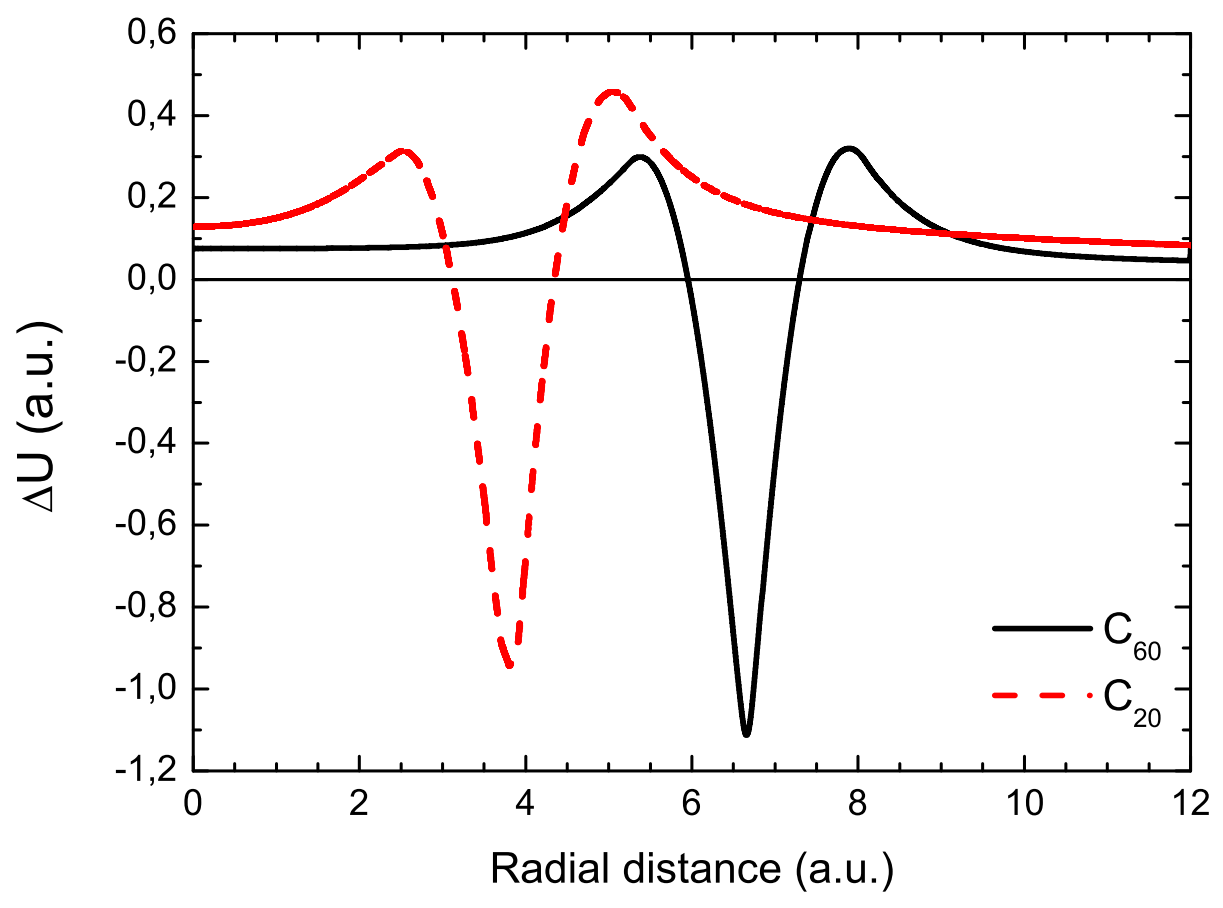

Figure 5. The additional pseudopotential $\Delta U$ in cases of $\mathrm{C}_{60}$ (solid curve) and $\mathrm{C}_{20}$ (dashed curve).

has a similar alternating-sign shape for both molecules but it is more asymmetric in the case of $\mathrm{C}_{20}$.

The single-electron energy spectra of $\mathrm{C}_{20}$ are presented in figure 6. The pseudopotential $\Delta U$ does not influence significantly on all nodeless orbitals while the single-node orbitals are shifted. This shift leads to a better agreement of the ab initio and jellium calculations for $2 \mathrm{~s}$ and $3 \mathrm{p}$ shells but gives a wrong value for the outer $4 \mathrm{~d}$ and $5 \mathrm{f}$ ionization potentials.

The additional pseudopotential $\Delta U$ exerts a similar influence on the electron density distribution of $\mathrm{C}_{20}$, as in case of $\mathrm{C}_{60}$ (see figure 7). In comparison with a standard jellium model (dashed red curve), the modified one improves the density distribution in the vicinity of the fullerene core (dash-dotted blue curve) but the electron density is spread partly to the spatial region $5-9$ a.u.

Having considered two different fullerenes within the spherical jellium model, one can conclude that the precise description of single-electron energy spectra of these systems by means of the jellium model is very difficult and elusive task, though such an approach produces mostly the right sequence of energy levels. Additional pseudopotentials allow one to obtain the right value of the ionization potential only for several outer shells but do not alter the overall situation significantly. At the same time, we suppose that by improving the ground-state density distribution with the introduced 


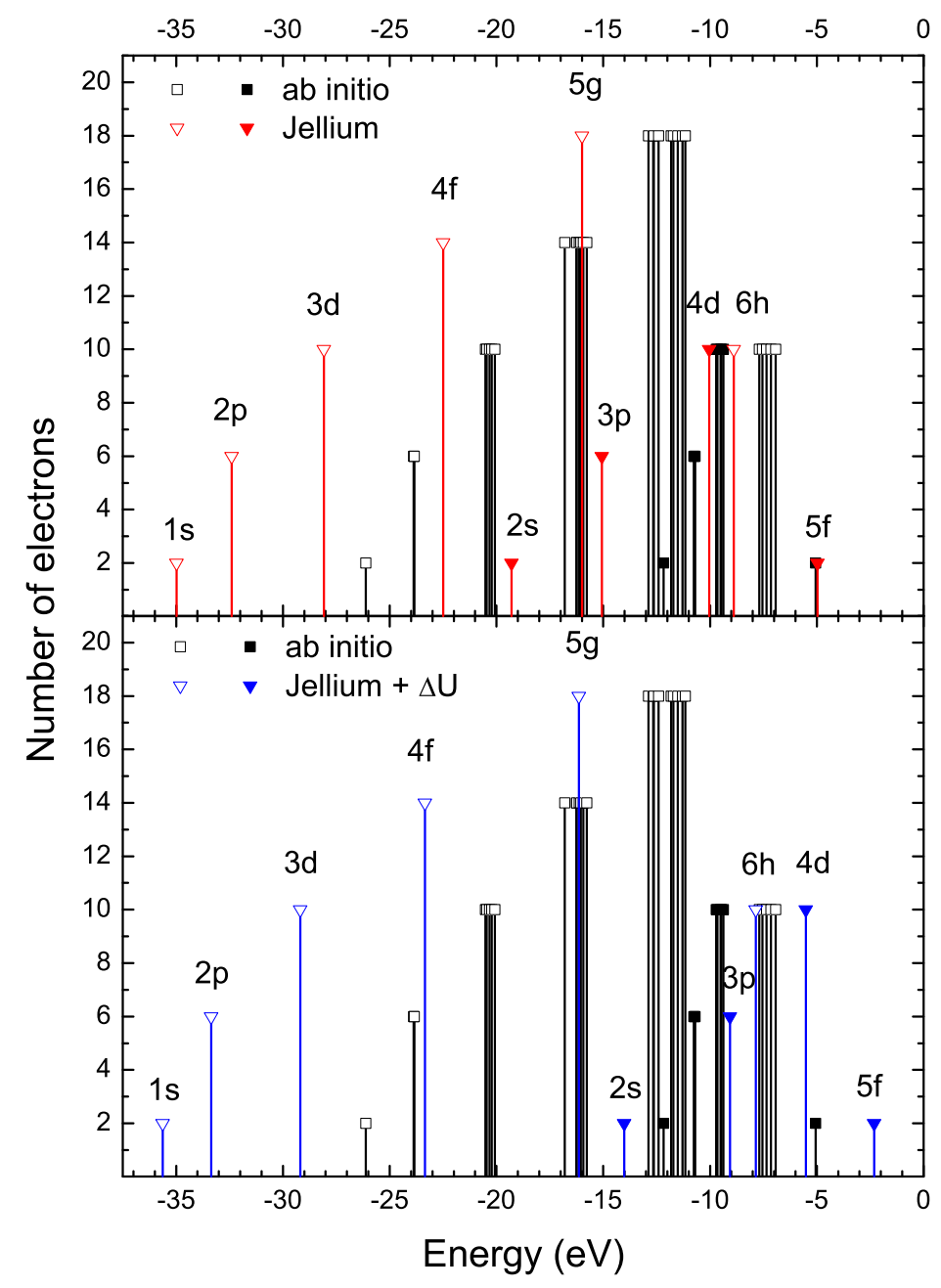

Figure 6. Single-electron energy levels of $\mathrm{C}_{20}$ obtained from the ab initio calculation (empty and filled squares) and within the jellium model (empty and filled triangles): the standard one (upper panel) and the modified one (lower panel). Nodeless $\sigma$-orbitals and single-node $\pi$-orbitals are labeled by empty and filled symbols, respectively.

pseudopotential one can achieve higher accuracy while constructing the photoionization amplitudes.

The obtained pseudopotentials for $\mathrm{C}_{60}$ and $\mathrm{C}_{20}$ can be well fitted by three Lorentz functions. The result of the fitting procedure is presented in figure 8 . Supposing $\Delta U(r) \equiv y(x)$, the resulting fitting function could be defined in the following form:

$$
y(x)=y_{0}+\sum_{i=1}^{3} \frac{2 A_{i}}{\pi} \frac{w_{i}}{4\left(x-x_{c_{i}}\right)^{2}+w_{i}^{2}},
$$

where $y_{0}$ is the offset constant, $x_{c}$ is the position of the peak maximum, $w$ is the fullwidth at half-maximum and $A$ is the normalization factor. The obtained values of these parameters are presented in table 1.

As was shown above, the pseudopotential $\Delta U$ has a more asymmetric form in the 


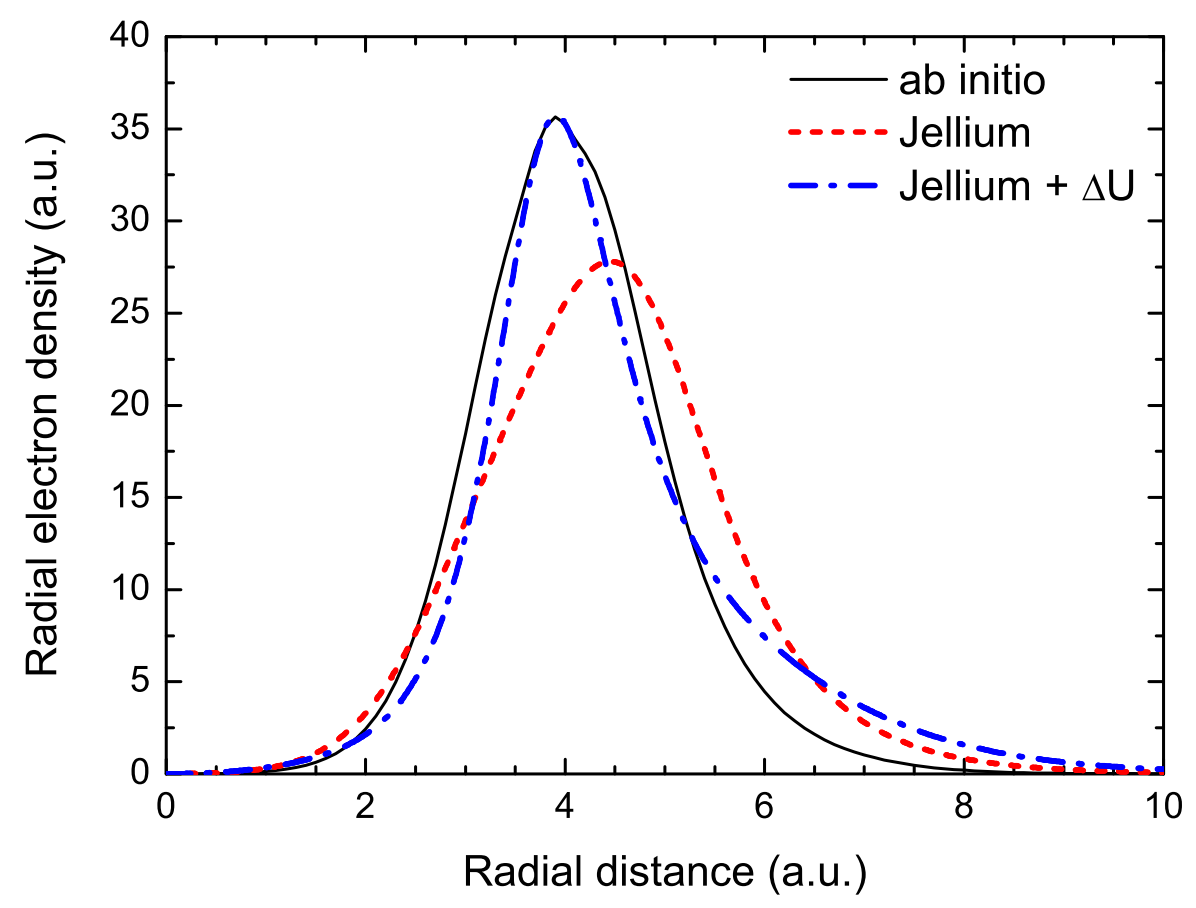

Figure 7. Radial electron density of $\mathrm{C}_{20}$ obtained from the ab initio calculation (solid curve) and calculated within the jellium model, the standard one (dashed curve) and with the additional pseudopotential $\Delta U$ (dash-dotted curve).

Table 1. Parameters of the Lorentz functions used for the fitting the pseudopotential $\Delta U$ for $\mathrm{C}_{60}$ and $\mathrm{C}_{20}$.

\begin{tabular}{|l|l|l|l|l|l|l|l|l|l|l|}
\hline & $y_{0}$ & $x_{c_{1}}$ & $w_{1}$ & $A_{1}$ & $x_{c_{2}}$ & $w_{2}$ & $A_{2}$ & $x_{c_{3}}$ & $w_{3}$ & $A_{3}$ \\
\hline $\mathrm{C}_{60}$ & 0.064 & 5.453 & 1.425 & 0.727 & 6.647 & 0.785 & -1.610 & 7.763 & 1.264 & 0.727 \\
$\mathrm{C}_{20}$ & 0.092 & 2.650 & 1.719 & 0.815 & 3.797 & 0.939 & -2.053 & 4.779 & 1.934 & 1.606 \\
\hline
\end{tabular}

case of $\mathrm{C}_{20}$ than in the case of $\mathrm{C}_{60}$; therefore, it should affect differently the $\pi$-electrons of these systems. Figure 9 represents the radial density of $\pi$-electrons in the $\mathrm{C}_{60}$ and $\mathrm{C}_{20}$ molecules obtained within the standard jellium model as well as the one augmented by $\Delta U$. The minimum of the $\pi$-electron density distribution is located at 6.78 a.u. for $\mathrm{C}_{60}$ and 4.03 a.u. for $\mathrm{C}_{20}$. These values are slightly shifted from the mean radius of the molecules, which equals 6.67 a.u. and 3.86 a.u., respectively. It is shown that due to the hybridization-related correction $\Delta U, \pi$-electrons in both systems are distributed non-uniformly in the inner and outer regions of the molecules.

To estimate a relative degree of spill-out of the $\pi$-electrons to the outer region of the fullerene molecules, we normalized the density distributions by dividing them by the number of the $\pi$-electrons in each system. We also shifted the $\pi$-electron density of $\mathrm{C}_{60}$ to the one of $\mathrm{C}_{20}$ to match the minima of the curves. The result is presented in figure 


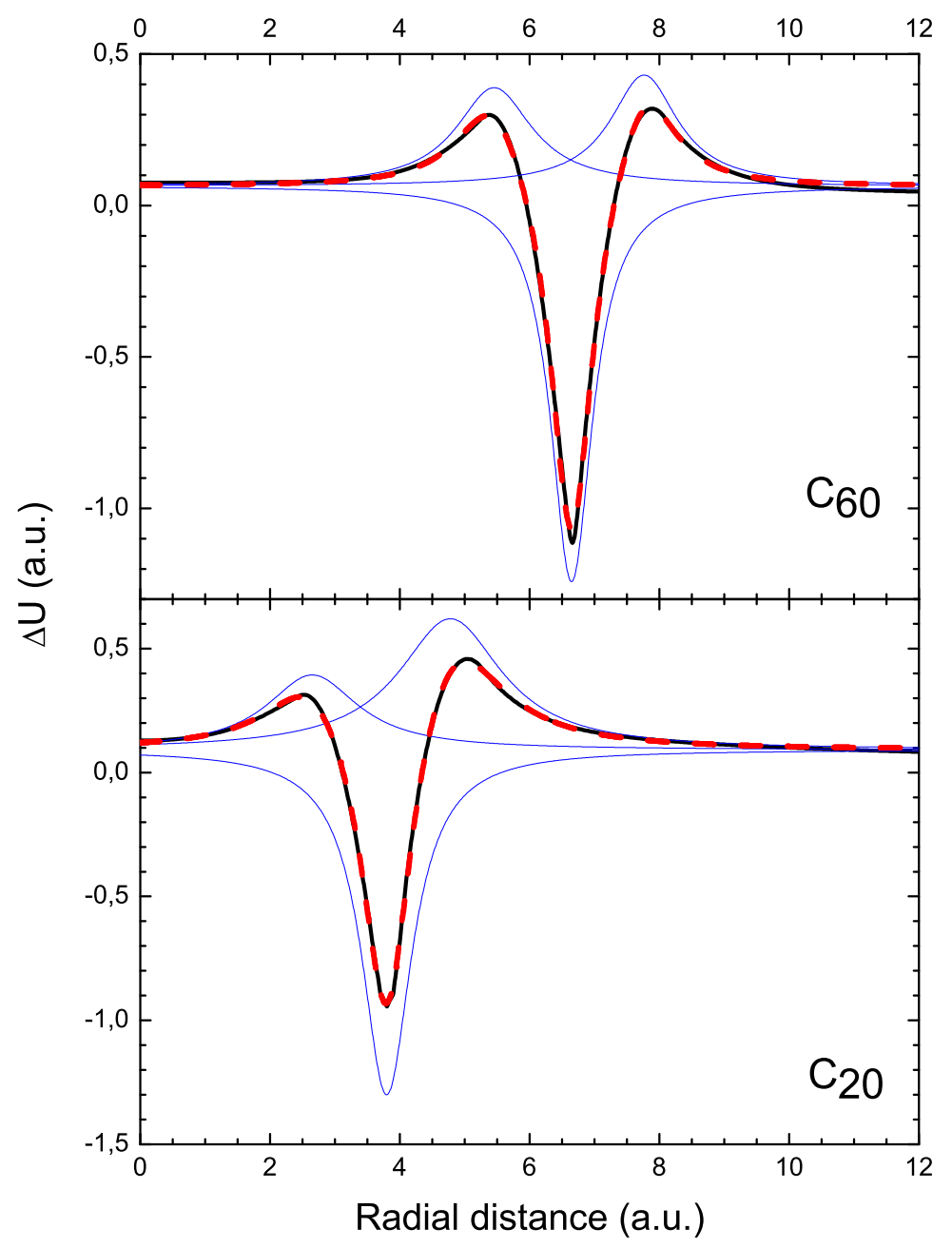

Figure 8. Pseudopotential $\Delta U$ for the $\mathrm{C}_{60}$ (upper panel) and $\mathrm{C}_{20}$ (lower panel) molecules. The initial curve is presented by the thick solid (black) line, dashed (red) line represents the fitting curve constructed as a sum of three primitive Lorentz functions (thin blue lines).

10. It is shown that the profile of the $\pi$-electron density in $\mathrm{C}_{60}$ differs from the one in $\mathrm{C}_{20}$. Due to the smaller radius of the molecule and a bigger curvature of the fullerene surface, $\pi$-electrons in $\mathrm{C}_{20}$ are spilled out further than in the case of $\mathrm{C}_{60}$. On the basis of this comparison, we suppose that for larger fullerenes, such as $\mathrm{C}_{240}, \pi$-electrons should be distributed more uniformly due to a smaller curvature of the surface of the molecule.

\section{Conclusion}

To conclude, we have introduced a new type of correction for description of the fullerenes $\mathrm{C}_{60}$ and $\mathrm{C}_{20}$ within the spherically symmetric jellium model. The correction is represented as an additional pseudopotential which originates from the difference between the precise $a b$ initio calculation and the one within the jellium model. Due to 


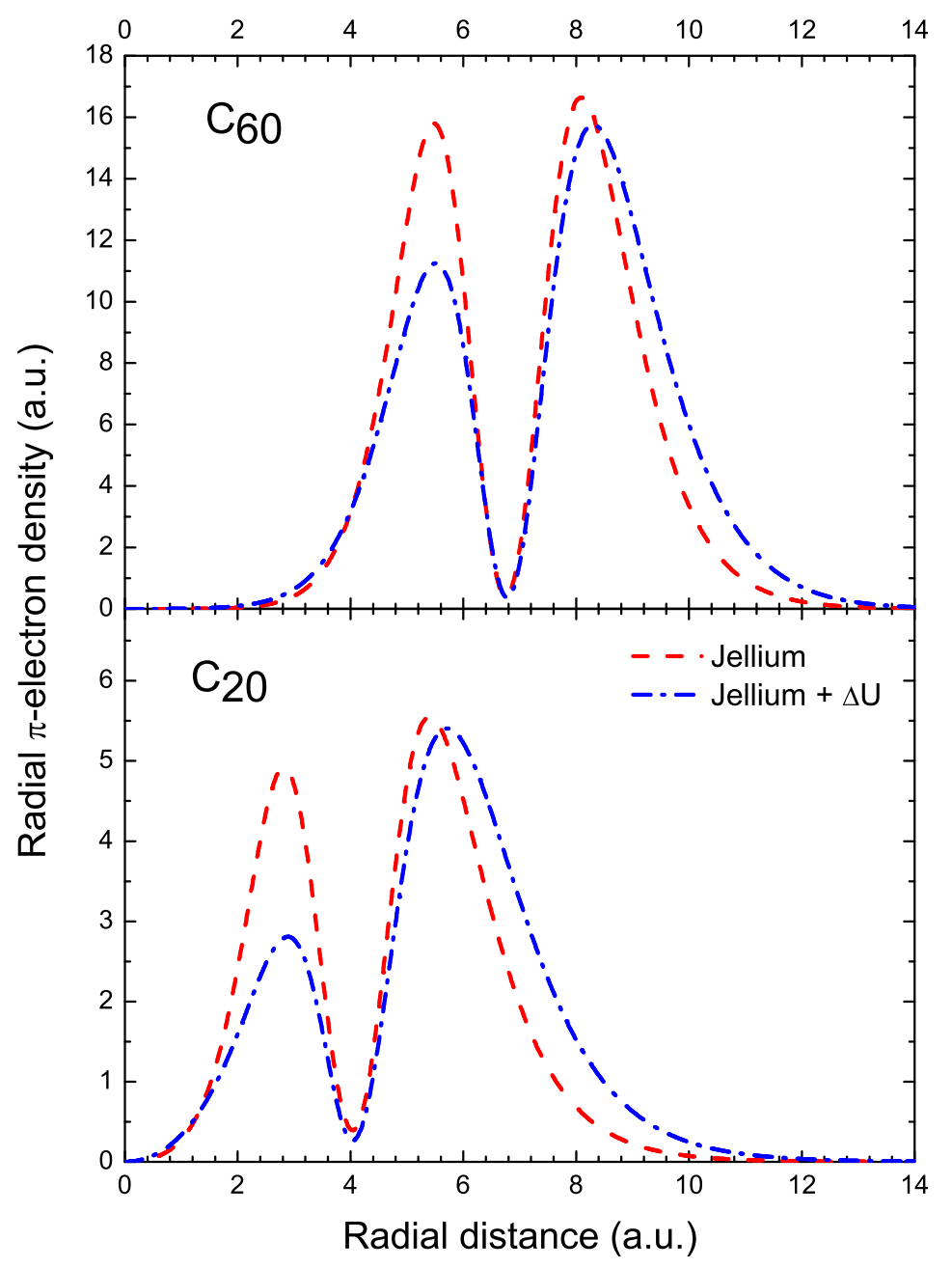

Figure 9. Radial density of $\pi$-electrons in $\mathrm{C}_{60}$ (upper panel) and $\mathrm{C}_{20}$ (lower panel) calculated within the standard jellium model (dashed red curve) and the modified jellium model with the presence of $\Delta U$ (dash-dotted blue curve).

the alternating-sign shape of the potential, it affects the $\sigma$ - and $\pi$-electrons of the system differently. Therefore, this potential allows one to mimic partially the $\mathrm{sp}^{2}$-hybridization, which occurs in formation of fullerenes, and, thus, to import the hybridization effects into the standard jellium model. We have shown that the correction used improves significantly the electron density distribution as compared to the standard jellium model and the one with an additional square-well pseudopotential. Like the other previously used corrections, it does not allow one to obtain a quantitative agreement with an $a b$ initio calculation for the single-electron energy spectrum but reproduces the sequence of energy levels corresponding to the one following from the more precise quantum-chemical calculation.

As the next step of this work, the correction to the jellium model, introduced in this paper, will be utilized further for the calculation of the dynamic response of fullerenes in 


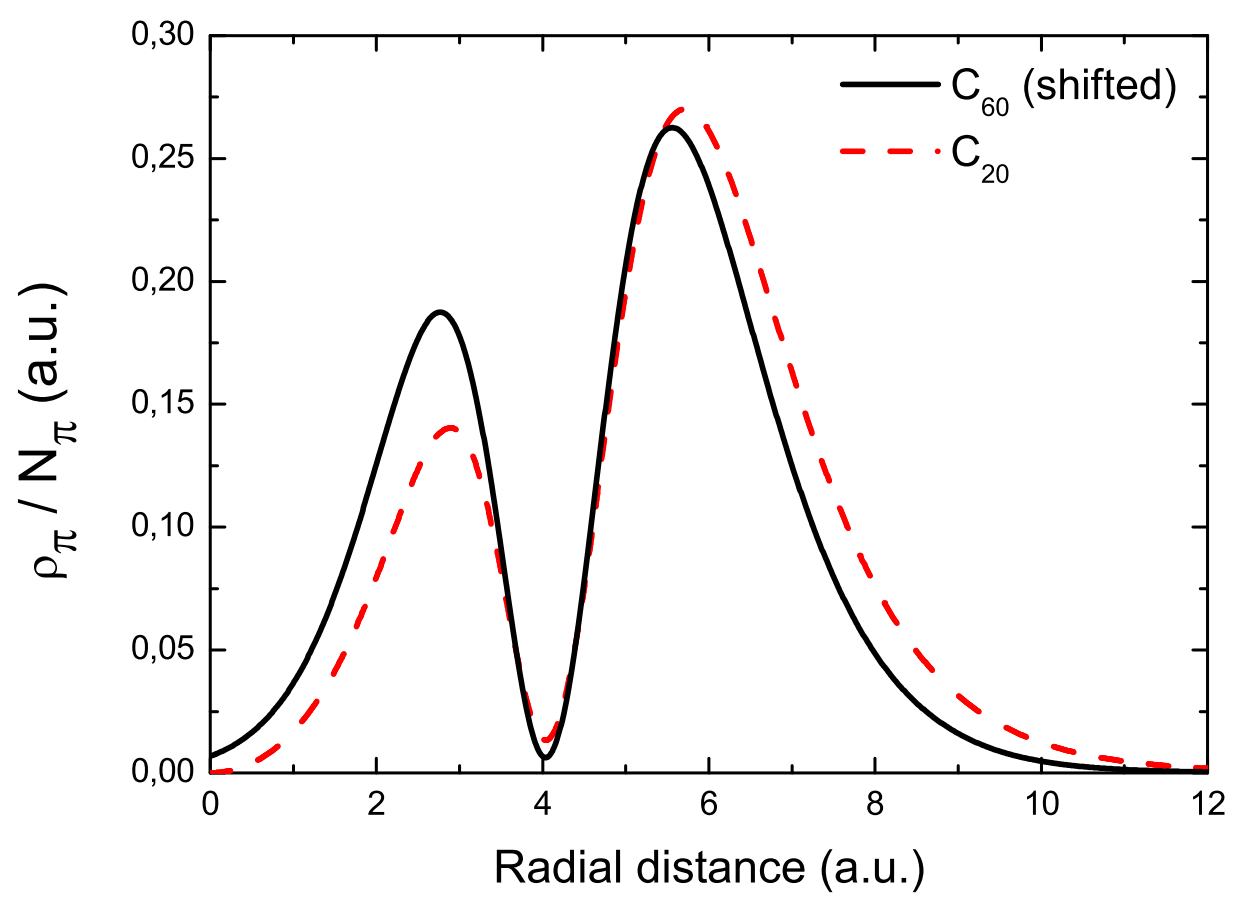

Figure 10. Radial $\pi$-electron densities, $\rho_{\pi}$, of the $\mathrm{C}_{60}$ and $\mathrm{C}_{20}$ fullerenes normalized by the number of the $\pi$-electrons, $N_{\pi}$, in each system. The density distribution of $\mathrm{C}_{60}$ is shifted to match the minima of the two curves (see the text for more explanation).

the processes of photon and electron impact excitation. We suppose that improving the ground-state density distribution with the introduced pseudopotential it is possible to get an accurate description of the excitation processes of fullerenes. Particular attention will be paid on the study of collective electron excitations. This work is currently in progress and the results will be presented elsewhere. An implementation of the presented formalism for larger fullerene molecules, nanotubes etc. could be another topic of further investigations.

\section{Acknowledgements}

A.V.V. is grateful to Deutscher Akademischer Austauschdienst (DAAD) for the financial support. The authors acknowledge the Frankfurt Center for Scientific Computing for the opportunity of carrying out complex ab initio calculations. R.G.P. and V.K.I. thank Frankfurt Institute for Advanced Studies for hospitality and acknowledge support from Russian Federal Program 'Scientific and Educational Manpower for Innovative Russia' (grant No. 2012-1.5-12-000-1011-007). 


\section{References}

[1] Kroto H W et al 1985 Nature $\mathbf{3 1 8} 162$

[2] Sattler K D 2010 Handbook of Nanophysics: Clusters and Fullerenes (Boca Raton: CRC Press)

[3] Mroz P et al 2008 Medicinal Chemistry and Pharmacological Potential of Fullerenes and Carbon Nanotubes vol 1, ed F Cataldo and T Da Ros (New York: Springer Science+Business Media B.V.) p 79

[4] Solov'yov A V 2005 Int. J. Mod. Phys. B 194143

[5] Frisch M J et al 2009 Gaussian 09 Revision A.1, Gaussian Inc. Wallingford CT

[6] Kaplan I G 1997 Z. Phys. D 40375

[7] Rubio A, Alonso J A, Blase X, Balbas L C and Louie S G 1996 Phys. Rev. Lett. 77247

[8] Solov'yov I A, Solov'yov A V and Greiner W 2004 J. Phys. B: At. Mol. Opt. Phys. 37 L137

[9] Ekardt W 1984 Phys. Rev. B 291558

[10] Kharchenko V A, Ivanov V K, Ipatov A N and Zhyzhin M L 1994 Phys. Rev. A 501459

[11] Polozkov R G, Ivanov V K, Verkhovtsev A V and Solov'yov A V 2009 Phys. Rev. A 79063203

[12] Yannouleas C and Landman U 1994 Chem. Phys. Lett. 217175

[13] Yabana K and Bertsch G F 1993 Phys. Scr. 48633

[14] Guet C and Johnson W R 1992 Phys. Rev. B 4511283

[15] Jänkälä K, Tchaplyguine M, Mikkelä M-H, Björneholm O and Huttula M 2011 Phys. Rev. Lett. 107183401

[16] Puska M J and Nieminen R M 1993 Phys. Rev. A 471181

[17] Wendin G and Wästberg B 1993 Phys. Rev. B 4814764

[18] Rüdel A, Hentges R, Becker U, Chakraborty H S, Madjet M E and Rost J-M 2002 Phys. Rev. Lett. 89125503

[19] Kidun O, Fominykh N and Berakdar J 2004 J. Phys. B: At. Mol. Opt. Phys. 37 L321

[20] Polozkov R G, Ivanov V K and Solov'yov A V 2005 J. Phys. B: At. Mol. Opt. Phys. 384341

[21] Madjet M E, Chakraborty H S, Rost J-M and Manson S T 2008 J. Phys. B: At. Mol. Opt. Phys. 41105101

[22] Pavlyukh Y and Berakdar J 2010 Phys. Rev. A 81042515

[23] Prinzbach H et al 2000 Nature 40760

[24] Zhang Q L et al 1986 J. Phys. Chem. 90525

[25] Krätschmer W, Fostiropoulos K and Huffman D R 1990 Chem. Phys. Lett. 170167

[26] Goroff N S 1996 Acc. Chem. Res. 2977

[27] Martins J L, Troullier N and Weaver J H 1991 Chem. Phys. Lett. 180457

[28] Haddon R C, Brus L E and Raghavachari K 1986 Chem. Phys. Lett. 125459

[29] Östling D, Apell P and Rosen A 1993 Europhys. Lett. 21539

[30] Gianturco F A, Kashenock G Yu, Lucchese R R and Sanna N 2002 J. Chem. Phys. 1162811

[31] Perdew J P and Zunger A 1980 Phys. Rev. B 235048

[32] Kohn W and Sham L J 1965 Phys. Rev. 140 A1133

[33] Lu T and Chen F 2012 J. Comp. Chem. 33580

[34] Lang N D and Kohn W 1970 Phys. Rev. B 14555

[35] Perdew J P and Monnierg R 1976 Phys. Rev. Lett. 371286

[36] Alasia F, Serra L, Broglia R A, Van Giai N, Lipparini E, Roman H E 1995 Phys. Rev. B 528488

[37] Vieira A, Begoña Torres M, Fiolhais C and Carlos Balbás L 1997 J. Phys. B: At. Mol. Opt. Phys. 303583 